\title{
A micro-architecture for binocular disparity and ocular dominance in visual cortex
}

\author{
Prakash Kara and Jamie D. Boyd \\ Department of Neurosciences, Medical University of South Carolina, Charleston SC 29425 USA
}

\begin{abstract}
In invertebrate predators like the praying mantis and vertebrate predators such as wild cats, the ability to detect small differences in inter-ocular retinal disparities is a critical means for accurately determining the depth of moving objects such as prey1. In mammals, the first neurons along the visual pathway that encode binocular disparities are found in the visual cortex. However, a precise functional architecture for binocular disparity has never been demonstrated in any species, and coarse maps for disparity have been found in only one primate species2,3. Moreover, the dominant approach for assaying the developmental plasticity of binocular cortical neurons employed monocular tests of ocular dominance to infer binocular function4. The few studies that examined the relationship between ocular dominance and binocular disparity of individual cells used single-unit recordings and have provided conflicting results as to whether ocular dominance can predict the selectivity or sensitivity to binocular disparity5-9. Here we use two-photon calcium imaging to sample the response to monocular and binocular visual stimuli from nearly every adjacent neuron in a small region of the cat visual cortex, area 18. We show that local circuits for ocular dominance always have smooth and graded transitions from one apparently monocular functional domain to an adjacent binocular region. Most unexpectedly, we discovered a new map in the cat visual cortex that had a precise functional micro-architecture for binocular disparity selectivity. At the level of single cells, ocular dominance was unrelated to binocular disparity selectivity or sensitivity. When the local maps for ocular dominance and binocular disparity both had measurable gradients at a given cortical site, the two gradient directions were orthogonal to each other. Together, these results suggest that from the perspective of the spiking activity of individual neurons, ocular dominance cannot predict binocular disparity tuning. However, the precise local arrangement of ocular dominance and binocular disparity maps provide new clues on how monocular and binocular depth cues may be combined and decoded.
\end{abstract}

Binocular vision and depth discrimination evolved more than 100 million years ago10. In mammals, the first single-cell description of a binocular disparity detector in the brain was made in the cerebral cortex of the cat approximately 40 years ago11. Numerous single-unit

\footnotetext{
Users may view, print, copy, and download text and data-mine the content in such documents, for the purposes of academic research, subject always to the full Conditions of use:http://www.nature.com/authors/editorial_policies/license.html\#terms

Correspondence and request for materials should be addressed to P.K. (kara@musc.edu).

Supplementary Information is linked to the online version of the paper at www.nature.com/nature.

Author contributions P.K. conceived the project, designed the experiments and setup the laboratory. P.K. and J.D.B. performed the experiments. P.K. analyzed the data and wrote the paper. Both authors discussed the results and commented on the manuscript.

Competing interests statement The authors declare that they have no competing financial interests.
} 
studies followed in both cats and macaque monkeys with pivotal electrophysiological and theoretical characterizations of the encoding of binocular disparity in the visual cortex, e.g., position vs. phase disparities and energy models12-15. In visual cortical neurons of mammals with frontally placed eyes, comparing the responses elicited by alternately stimulating each eye demonstrates the presence of a full range of ocular dominance, from completely contralateral through binocular to completely ipsilateral cells. A neuron tuned for binocular disparity, by definition, must receive visual input from both eyes. Therefore, it is reasonable to suppose that only binocular and not monocular cells would show robust disparity selectivity. A relationship is also suggested by misaligning the two eyes during the critical period of postnatal development. The misalignment leads to a loss of visual cortical neurons that can be driven through either eye16, i.e., neurons lose their ocular dominance and become ocular exclusive. The misalignment also leads to stereo blindness17. Although ocular dominance is among the premier models of postnatal developmental plasticity4, testing the input from each eye independently fails to show the suppressive effects or the summation of sub-threshold inputs that can code for disparity in "monocular" cortical cells18. Indeed, from single-unit electrophysiological studies, no consensus could be reached on the relationship between ocular dominance and binocular disparity5-9.

By assaying binocular disparity and ocular dominance for nearly every neuron in a local volume of cat visual cortex using calcium imaging, we examined whether there is an orderly representation of binocular disparity and ocular dominance, and whether these two features were inter-related at the level of single cells and the local map structure. Our calcium indicator loading protocol typically labelled several hundred adjacent cortical layer $2 / 3$ neurons in a spherical region (diameter 300-600 $\mu \mathrm{m}$ ). Two-photon calcium imaging then permitted the simultaneous measurement of the visual responses of 100-150 neurons within a single optical cross section parallel to the cortical surface. All $300 \times 300 \mu \mathrm{m}$ imaged sites were iso-orientation and iso-direction selective (Supplementary Figs. S1a-c and S5b). Since we had to perform a battery of tests for ocular dominance, disparity, orientation, direction, spatial frequency, and retinotopy, we did not probe disparity at orientation pinwheel sites. For our ocular dominance and binocular disparity measurements, we interleaved monocular and binocular drifting sine grating visual stimuli (see Fig. 1a). The two monocular stimuli and eight inter-ocular spatial phase disparity stimuli were always presented at the orientation and direction optimal for the imaged site (see Supplementary Figs. S1a-c and S5b). Twelve cats were used in this study. In the first three animals, only ocular dominance was assessed ( $n=857$ cells) to determine the long-term stability of monocular responses over time (Supplementary Fig. S1d-e). In seven subsequent animals, monocular stimuli were always interleaved with binocular disparity stimuli ( $n=2,028$ cells). In two additional animals, imaging with simultaneous electrophysiological controls was performed (Supplementary Fig. S2).

With calcium imaging, individual visual cortical neurons showed robust and highly reproducible trial-by-trial responses to monocular stimuli and binocular disparity stimuli. Figure $1 \mathrm{~b}$ shows the time course of the calcium indicator fluorescence signal evoked by visual stimulation for five simultaneously recorded cells from a single animal. Cell 1 had near equal responses to either monocular visual stimulus, robust responses to five of the 
eight presented disparity stimuli, and a clear suppression of visual responses to at least three binocular disparity stimuli $\left(45^{\circ}, 90^{\circ}\right.$, and $135^{\circ}$ inter-ocular spatial phase disparities). Cell 3 responded almost exclusively to stimulation of the left eye when probed with monocular visual stimuli but showed a profound modulation to binocular disparity stimulation with a peak response at $135^{\circ}$ inter-ocular spatial phase disparity. Cell 4 had weak responses to monocular stimuli but again displayed potent modulation to specific phases of binocular disparity stimuli. Cell 5 was narrowly tuned to respond to a binocular spatial phase disparity of $0^{\circ}$.

The diversity of disparity tuning across neighbouring cells from a single $300 \times 300 \mu \mathrm{m}$ imaged site (e.g., Fig. 1b) might imply the lack of a locally organized map for disparity. However, the cell-based disparity map (Fig. 1d) showed a smooth progression of preferred disparity phase from the bottom to the top of the imaged site. Only cells significantly tuned (selective) for binocular disparity phase were colour-coded in Fig. 1d and all subsequent cell-based disparity maps. For the data shown in Fig. 1d, of the 140 cells identified, 96\% were significantly responsive to disparity stimuli $(P<0.05$, ANOVA across blank and 8 disparity periods) and $85 \%$ were tuned to binocular disparity $(P<0.05$, ANOVA across 8 disparity periods). In all colour-coded disparity phase maps, $0^{\circ}$ preferred phase did not necessarily correspond to $0^{\circ}$ absolute disparity. As demonstrated in previous studies in anesthetized cats and monkeys18-21, varying relative spatial phase disparity with sine gratings provides robust indices of disparity selectivity and sensitivity (also see Supplementary Discussion). The map for ocular dominance (Fig. 1e) from the same site as shown in Figure 1d was relatively pure with virtually all cells being binocular with a slight contralateral bias, as confirmed in the ocular dominance histogram (Fig. 1f). Data from another cat are shown in Figure 1g-k. The responses from five individual cells to monocular stimuli and binocular disparity stimuli were once again very robust (Fig. 1g). However, at this site, monocular stimulation evoked responses almost exclusively from one eye (ipsilateral). Nevertheless, binocular disparity stimuli evoked significant and selective modulation of responses. The disparity map corresponding to this second site also showed a smooth transition of preferred disparity across the imaged area (Fig. 1i). As expected from the time courses shown in Fig. 1g, the ocular dominance map and histogram showed a strong bias to ipsilateral eye stimulation (Fig. $1 \mathrm{j}-\mathrm{k}$ ).

The two experiments described in Figure 1 each have regions with a very narrow range of ocular dominance preferences. That a strong map for disparity phase is present under both conditions suggests that disparity phase may be insensitive to ocular dominance. In sixteen $300 \times 300 \mu \mathrm{m}$ imaged areas from eleven calcium indicator dye injection sites in seven animals, not a single site showed a significant correlation between the cells' preferred disparity phase and their ocular dominance ( $R=0.001$ to $0.181 ; P=0.07$ to 0.99 per imaged area). The monocularity index 9 , which ignores the sign of ocular dominance (ipsi vs. contra) and quantifies only the strength of eye dominance, also did not yield a significant correlation with the cells' preferred disparity phase $(R=0.001$ to $0.204 ; P=0.06$ to 0.99 per imaged area).

The independence of preferred disparity phase from ocular dominance at the level of single cells is best demonstrated when an individual $300 \times 300 \mu \mathrm{m}$ imaged site contained cells that 
had the full range of ocular dominance indices (Fig. 2). From qualitative observation of the cell-based maps for preferred disparity phase and ocular dominance (Fig. 2a-b), they appeared to be orthogonally oriented (white and black arrowheads). We quantified the relative gradient direction of these maps by first smoothing the raw pixel maps for preferred disparity and ocular dominance (Fig. 2d-e). Smooth pixel maps were also derived from cellbased maps (see Full Methods) and produced virtually identical results. The relative gradient direction of the ocular dominance and disparity maps was even more apparent when the disparity and ocular dominance maps were overlaid as contour plots (Fig. 2f). The relative gradient direction of the two maps was quantified by calculating the pixel-by-pixel difference in gradient direction for the two maps (Fig. 2g, Supplementary Figs. S3, S4, and Full Methods). A histogram of the distribution of the gradient direction difference for the two maps shows a clear peak near $90^{\circ}$, confirming that the maps were near perfectly orthogonal (also see Supplementary Fig. S3). From the fitted curve (red, Fig. 2g), we first calculated the ratio of the peak to the baseline (VMratio - see Full Methods). Per imaged site, a VMratio $>3$ was considered to represent a significant interaction of the two maps ( $n=$ $8 / 16$ imaged areas, each $300 \times 300 \mu \mathrm{m}$, showed significant interaction). The other eight imaged areas had no significant interaction $($ VMratio < 2). The lack of map interaction was further confirmed by randomizing the pixels in one of the two maps and showing that the gradient direction difference histograms still had a VMratio of less than 2. In the imaged areas $(300 \times 300 \mu \mathrm{m})$ where VMratio was less than 2, the gradient direction difference histograms from randomized vs. non-randomized maps were statistically indistinguishable ( $P=0.404-0.867, Z=0.16-0.83$, Sign test). Thus, if the VMratio was $<2$, no interaction can be determined between two maps. Several examples of imaged sites that had either significant or no interaction are shown in Supplementary Fig. S4. The VMratio was correlated with the ocular dominance variance of cells ( $\sigma 2$ cells) per imaged site $(R=0.65$; $P<0.01 ; n=16$ imaged areas). Thus, significant interaction of the disparity phase and ocular dominance maps was more likely when the full range of ocular dominance was represented at a given site (e.g., Fig. 2) compared to when a narrow range of ocular dominance was represented per site (e.g., both cases in Fig. 1). We define "orthogonality" as an angle difference in the range between $45-135^{\circ}$. For the eight $300 \times 300 \mu \mathrm{m}$ imaged areas that showed a significant ocular dominance vs. disparity phase gradient interaction, the peak relative direction of the two gradients had a median angle of $85^{\circ}$ with $25 \%$ and $75 \%$ quartile ranges falling within $66-99^{\circ}$ (Fig. $2 \mathrm{~h}$, peak calculated from red curve fitted to histogram). Additional statistics on map interaction, e.g., Median Scalar Product, are given in the Supplementary Discussion.

Having shown that preferred binocular disparity phase is not related to ocular dominance at the level of single cells, we tested the possibility that ocular dominance might predict the sensitivity to binocular disparity. From qualitative observations, sites dominated by responses to monocular stimulation of either eye and sites responsive to monocular stimulation of only one eye appear to be just as likely to show robust disparity tuning. In the three imaged sites shown in Figures 1 and 2, 80-99\% of cells were responsive and 80-92\% of cells were selective for binocular disparity. Across all experiments in seven animals, 75\% $(1,512 / 2,028)$ of cells were responsive to disparity stimuli. Of these visually responsive cells, $73 \%(1,097 / 1,512)$ were tuned for binocular disparity. Thus, some individual $300 \times$ 
$300 \mu \mathrm{m}$ imaged sites only had $\sim 30 \%$ of cells tuned for disparity. Furthermore, only $5 \%$ of all cells $(101 / 2,028)$ were truly monocular, i.e., responsive to stimulation of one eye and not significantly responsive to disparity stimulation. To explicitly determine whether ocular dominance influenced the sensitivity to binocular disparity stimuli across our entire sample, we only considered cells that were significantly responsive to binocular disparity and monocular stimuli ( $n=1,119$ cells). Disparity sensitivity is reflected in the entire tuning curve for disparity, including facilitation and suppression relative to the mean response (Fig. 3a). We established that the ratio of the amplitude of a sine-fitted tuning curve for disparity to the mean response $(\mathrm{F} 1 / \mathrm{F} 0)$ was a reliable index of disparity sensitivity (Supplementary Fig. S6) and found that disparity sensitivity and ocular dominance were uncorrelated (Fig. 3b, $R=0.041, P=0.170)$. F1/F0 was larger in sites where a significant map interaction between disparity and ocular dominance was measured (VMratio $>3$ ), compared to sites where no map interaction was detected (VMratio <2), i.e., F1/F0 $=0.653 \pm 0.011$ vs. 0.485 $\pm 0.009, \mathrm{P}<0.00001, \mathrm{~T}$ test). But an F1/F0 of $\sim 0.5$ still represents very potent modulation.

Binocular disparity maps from a single injection site were stable over protracted time periods, up to the 12-hour maximum time we recorded from some sites (Fig. 4). The smoothness of the transition from one disparity domain to the next can best be appreciated from pixel maps (bottom row in Fig. 4). In these pixel maps, cell boundaries were ignored; the hue of each pixel was determined by the best disparity; and the brightness of each pixel was determined by the magnitude of the response vector. Such pixel maps therefore represent the combined response from cell bodies and surrounding neuropil22. The preferred disparity at all three depths changed systematically from the bottom right to the top left of each area and the mean disparity gradient was indistinguishable for the three depths (Fig. 4).

The trial-by-trial stability of responses reflected in the time course of fluorescence changes to disparity stimuli, stable monocular retinotopy measurements (Supplementary Fig. S5), and the stability of the disparity maps over 12 hours of recording suggests that we had no artefacts from eye movements or drift in our anesthetized and paralyzed animals. Small spatial frequency gradients were occasionally present in our imaged sites. However, they were orthogonal to the binocular disparity gradient (Supplementary Fig. S3). Imaging and electrophysiological controls suggested that changes in calcium fluorescence were not saturating and matched the spiking activity in individual cells (Supplementary Fig. S2). Additional analytical controls confirmed that any potential response onset transients did not confound our disparity tuning measurements at the level of single cells and the overall map structure (Supplementary Fig. S7).

Since we simultaneously recorded from at least 100 cells per given site in cortical layer $2 / 3$ with no sampling bias, it is unlikely that we missed an otherwise true correlation between ocular dominance and preferred binocular disparity phase or binocular disparity sensitivity. Perhaps for simple cells in the primary recipient zone of thalamic input (cortical layer 4), a correlation between ocular dominance and binocular disparity will be found. However, complex cells in cat layer $2 / 3$ are more ideally suited for disparity detection than simple cells 23 and the overwhelming majority of our cells in layer $2 / 3$ were binocular when probed with disparity stimuli. 
The existence of a map for binocular disparity in area 18 of the cat visual cortex revealed with two-photon calcium imaging suggests that disparity maps may be more common across species with frontally placed eyes than previously thought. Individual iso-disparity domains in macaque extra-striate areas V2 and MT can be relatively large (750-1,500 $\mu \mathrm{m})$, resulting in readily detectable maps for disparity with microelectrode or intrinsic imaging techniques2,3. For ocular dominance maps, under no circumstances did we observe fractures (or jumps) in the map from ispilateral to contralateral eye dominated regions. Transitions from binocular to apparently 'monocular' ocular dominance domains were always smooth. This suggests that the apparently weaker map structure for ocular dominance seen with conventional optical imaging methods 24 does not result from local mixing of neurons that have different ocular dominance indices.

The most comprehensive single-unit study to date in primate V1 did show independence between binocular disparity and ocular dominance at the level of single cells9. However, our two-photon calcium imaging experiments crystallize the exact relationship in the cat visual cortex by showing that the independence of disparity and ocular dominance at the level of single cells does not arise from a local salt-and-pepper arrangement of maps for either disparity or ocular dominance. From a developmental standpoint, a map for ocular dominance may initially reflect residual imbalances in the density of inputs from each eye25. However, a smooth map for ocular dominance may serve as a scaffold for the formation of disparity maps. Neurons embedded in local cortical regions where preferred disparity is organized in a map may be more sensitive to binocular disparity compared to adjacent regions that are less well organized, as is evident in primate MT2. A potential computational advantage of the relationship between disparity and ocular dominance maps for binocular visual processing is that a wide range of disparity encoding is maintained independent of local changes in ocular dominance. Locally organized ocular dominance and binocular disparity maps might optimize the processing of multiple depth cues by maximizing the coverage of binocular disparity and occlusion cues from surfaces located at different depths for which the 'eye of origin' needs to be known26. Cortical neurons tuned to this combination of features respond vigorously to monocular stimulation of one eye only but still show modest disparity tuning, e.g., see cells 18, 58, 66, 71, 86, 95, and 106 in Supplementary Fig. S6b. Future studies could determine whether locally organized maps for ocular dominance and disparity play a role in speeding-up the decoding (or readout) of these combined cues by other visual cortical areas. While local orthogonality is an emergent property when multiple overlapping functional maps are simulated in the general class of self-organizing or dimension reduction models27-29, it remains to be determined whether ocular dominance and disparity maps conform to various predictions made from such models.

\section{METHODS SUMMARY}

Cats (postnatal days 36-49) were anesthetized with isoflurane (1-2\% in surgery, $0.5-1.0 \%$ during imaging) 22 and paralyzed with vecuronium bromide 22 . A craniotomy was performed over area 18 of the visual cortex, the dura reflected, and the underlying cortex covered with agarose. Movement of the brain from respiratory and heart beat pulsations were negligible (Supplementary Fig. S8). The cell-permeant calcium indicator Oregon Green 488 Bapta-1 
AM $(1 \mathrm{mM})$ was prepared22,30 and co-loaded with $40 \mu \mathrm{M}$ Alexa Fluor 594 into a glass patch pipette ( $2.5 \mu \mathrm{m}$ diameter tip). Under continuous visual guidance, the pipette tip was advanced 200-250 $\mu \mathrm{m}$ below the cortical surface and the indicators were then pressure ejected (5-10 psi). This particular method of loading produces minimal staining of glial cells (see Ref 22) but it is possible that some of the stained cells in the present study were not neuronal. Fluorescence was monitored with a custom-built microscope (Prairie Technologies) coupled with a Mai Tai XF (Newport Spectra-Physics) mode-locked Ti:sapphire laser ( 850 or $920 \mathrm{~nm}$ ). Drifting sine-wave gratings ( $2 \mathrm{~Hz}, 50 \%$ contrast) were presented on a CRT (100 Hz refresh rate) in a variety of configurations for orientation, direction of motion, spatial frequency, ocularity (left or right eye-for ocular dominance), and eight inter-ocular spatial phase disparities $\left(0^{\circ}, 45^{\circ}, 90^{\circ}, 135^{\circ}, 180^{\circ}, 225^{\circ}, 270^{\circ}, 315^{\circ}\right)$. For ocular dominance and binocular disparity assays, animals viewed the monoptic and dichoptic visual stimuli through ultra-fast ferroelectric liquid crystal shutters $(7 \mathrm{KHz}$ switching time, 1,000:1 extinction contrast ratio, DisplayTech). Each stimulus period ( $8 \mathrm{~s}$ ) was preceded by an equal blank period, repeated 3-8 times. Coarse retinotopic positions of monocular receptive fields were determined by using $5^{\circ}$ wide flashing bars of light or strips of gratings at ten retinotopic positions. Two-photon images were analyzed inMatlab (Mathworks)—see Full Methods.

Full Methods and any associated references are available in the online version of the paper at www.nature.com/nature.

\section{METHODS}

Images were analyzed using customized Matlab (Mathworks) software. Cells were identified through a series of morphological filters that defined the contours of cell bodies based on intensity, size, and shape22. Time courses of individual cells were extracted by calculating mean pixel values within cell contours 22 . Visually responsive cells were defined by ANOVA across blank and $N$ test visual stimuli $(P<0.05)$. Cells selective for particular stimuli were defined by ANOVA across $N$ stimulus periods $(P<0.05)$.

Ocular dominance (OD) was derived from the responses to monocular stimulation and defined as:

$$
O D=\frac{R_{i p s i}}{\left(R_{\text {ipsi }}+R_{\text {contra }}\right)}
$$

Sensitivity to binocular disparity (F1/F0) was derived by vector averaging as follows:

$$
\vec{V}_{\text {avg }}=\sum_{i=1}^{n} \frac{\vec{V}_{i}}{n}
$$

Where $V \rightarrow_{i}$ is a vector with direction equal to disparity phase, length equal to the corresponding cell's response amplitude and $n$ is the total number of disparity phases. 
The average amplitude of the response to disparity stimulation was defined as:

$$
A_{a v g}=\sum_{i=1}^{n} \frac{\left|\vec{V}_{i}\right|}{n}
$$

Then F0 and F1 were calculated as:

$$
\begin{gathered}
F 0=A_{\text {avg }} \\
F 1=2\left|\vec{V}_{\text {avg }}\right|
\end{gathered}
$$

The direction of $V \rightarrow_{\text {avg }}$ provided the phase of the best response to disparity stimulation.

Due to the low trial-by-trial variability of our data coupled with the use of sinusoidal grating visual stimuli, F1/F0 was a reliable index of sensitivity to disparity, as confirmed with Monte Carlo-derived estimates of standard deviation of fit parameters and coefficients of determination $\left(\mathrm{R}^{2}\right)$.

Each time we calculated an analytical fit of experimental data we conducted Monte Carlo simulations (128 trials) to estimate the error of these analytical fits. For each Monte Carlo trial, we randomly modified values assuming they had Gaussian distributions with standard error as calculated from the analysis of the experimental data. Analytical fits were done for each simulated data set and the mean was calculated for all Monte Carlo trials. In all cases, the Monte Carlo derived means were nearly identical to the original data fit and we used the Monte Carlo derived standard deviations as error estimates of the fitting procedure. If cells passed the experimental alpha criteria for disparity selectivity $(P<0.05$, ANOVA), then the mean F1/F0 was at least twice larger than the standard deviation derived from the Monte Carlo simulations (Supplementary Fig. S6c). For monocular retinotopy experiments, the Monte Carlo derived standard deviations were used to determine which experiments had retinotopic measures that were sufficiently reliable to use as an index of vergence state (see Supplementary Discussion).

To quantify the relative gradient direction of two maps, e.g., disparity phase and ocular dominance, we first calculated the pixel-by-pixel gradient of smoothed pixel maps (cf. cellbased maps, below). We used a built-in Matlab function where the gradient of a function of two variables $\mathrm{F}(\mathrm{x}, \mathrm{y})$ was defined as:

$$
\nabla F=\frac{\partial F}{\partial x} \hat{i}+\frac{\partial F}{\partial y} \hat{j}
$$

To capture the global relationship between the two maps that have cellular structure, it was necessary to smooth the maps with a filter that is larger than the distance between two cells. Thus, each map was first lowpass Gaussian filtered with a standard deviation of 50 pixels (30 $\mu \mathrm{m}$ for $300 \times 300 \mu \mathrm{m}$ imaged regions). To remove small filtering artefacts present at edges (see Supplementary Fig. S9), borders around each map were excluded ( $52 \mu \mathrm{m}$ on each 
side of $300 \times 300 \mu \mathrm{m}$ imaged regions; $105 \mu \mathrm{m}$ on each side of $600 \times 600 \mu \mathrm{m}$ imaged regions).

For ocular dominance maps, the Gaussian filter was applied directly to pixel values of the ocular dominance map. Since disparity is a circular variable, an alternative smoothing procedure was used for the disparity phase map. First, two separate component maps (sine and cosine) were generated from the disparity angle map. Each component map was smoothed by the Gaussian filter. Then each of the two smoothed component maps was combined back to a single disparity angle map.

To conduct an equivalent gradient analysis on cell-based maps, we first transformed cellbased maps to pixel maps as follows: We derived a value for each pixel $P_{x, y}$ byinterpolating corresponding values from all cells surrounding each pixel. The interpolation was a weighted mean, where each weight was calculated as a Gaussian function of the distance to each cell:

$$
P_{x, y}=\frac{\sum_{\text {cell }=1}^{n} P_{\text {cell }} W\left(X_{\text {cell }}, Y_{\text {cell }}, X, Y\right)}{\sum_{\text {cell=1 }}^{n} W\left(X_{\text {cell }}, Y_{\text {cell }}, X, Y\right)}
$$

Where:

$P_{x, y}=$ new pixel value (disparity phase, ocular dominance) at each $\mathrm{x}, \mathrm{y}$ coordinate in the map

$P_{c e l l}=$ corresponding cell-based value (disparity phase, ocular dominance)

$W=$ Gaussian function

To maintain consistency with the Gaussian lowpass filter we used to smooth raw pixel maps, the standard deviation of the Gaussian function for the pixel maps used here was 50 pixels, which corresponds to $30 \mu \mathrm{m}$ (for $300 \times 300 \mu \mathrm{m}$ imaged areas). Once pixel maps were generated from cell-based maps, the procedures for smoothing were identical as described earlier for raw pixel-based maps.

The gradient direction difference for two maps was calculated using the built-in Matlab function $\nabla F$, as described above. Since we were only interested in the relative direction of two simultaneously recorded maps, e.g., disparity phase and ocular dominance, the gradient direction difference was collapsed to a $0-180^{\circ}$ range (Fig. $2 \mathrm{~g}$ ). Each histogram was 36 bins in length and each bin represented 5 degrees.

To quantify the gradient direction difference distribution, we conducted two independent analyses of these histograms. First, using a least-squares method, we fit a von Mises function to the histogram:

$$
G=A_{\min }+A_{1} \exp \left\{A 2\left(\cos \left[\left(D \operatorname{dir}-D \operatorname{dir}_{0}\right) \frac{\pi}{90}\right]-1\right)\right\}
$$


Where $A_{\min }$ is the value of the smallest bin in the distribution, and $A_{1}, A_{2}, D d i r_{0}$ are fitting parameters.

The ratio of the maximum to the minimum of the fitted function (VMratio) was the first metric we used to quantify strength of the interaction of the two maps:

$$
\text { VMratio }=\frac{G_{\max }}{G_{\min }}
$$

The second metric of the gradient direction difference histogram was calculated as the ratio of the number of pixels in 9 bins around the peak bin (Max bin \pm 4 ) to the total number of analyzed pixels:

$$
\text { Bin ratio }=\frac{\sum_{N_{\max }+4}^{N_{\max }+4} N_{n}}{\sum_{1}^{36} N_{n}}
$$

Where $N_{n}$ is the value of bin number $n$.

Pooling data from all imaged sites, these two measures of the strength of the mapinteraction were correlated $(R=0.89 ; P<0.00001)$.

\section{Supplementary Material}

Refer to Web version on PubMed Central for supplementary material.

\section{Acknowledgments}

We thank B. Cumming and N. Swindale for helpful discussions. We thank B. Shi, Z. Shen, Z. Lu, and J. Schnellmann for comments on the manuscript. This work was supported by grants from the NIH, Whitehall and Dana Foundations to P.K.

\section{References}

1. Rossel S. Binocular stereopsis in an insect. Nature. 1983; 302:821-822.

2. DeAngelis GC, Newsome WT. Organization of disparity-selective neurons in macaque area MT. J Neurosci. 1999; 19:1398-1415. [PubMed: 9952417]

3. Chen G, Lu HD, Roe AW. A map for horizontal disparity in monkey V2. Neuron. 2008; 58:442450. [PubMed: 18466753]

4. Hubel DH, Wiesel TN. Early exploration of the visual cortex. Neuron. 1998; 20:401-412. [PubMed: 9539118]

5. Poggio GF, Fischer B. Binocular interaction and depth sensitivity in striate and prestriate cortex of behaving rhesus monkey. J Neurophysiol. 1977; 40:1392-1405. [PubMed: 411898]

6. Ferster D. A comparison of binocular depth mechanisms in areas 17 and 18 of the cat visual cortex. J Physiol (Lond). 1981; 311:623-655. [PubMed: 7264985]

7. Gardner JC, Raiten EJ. Ocular dominance and disparity-sensitivity: why there are cells in the visual cortex driven unequally by the two eyes. Exp Brain Res. 1986; 64:505-514. [PubMed: 3803488]

8. LeVay S, Voigt T. Ocular dominance and disparity coding in cat visual cortex. Vis Neurosci. 1988; 1:395-414. [PubMed: 3154808]

9. Read JC, Cumming BG. Ocular dominance predicts neither strength nor class of disparity selectivity with random-dot stimuli in primate V1. J Neurophysiol. 2004; 91:1271-1281. [PubMed: 14523074] 
10. Parker, A. In the blink of an eye: how vision sparked the big bang of evolution. Basic Books; New York: 2003.

11. Barlow HB, Blakemore C, Pettigrew JD. The neural mechanism of binocular depth discrimination. J Physiol (Lond). 1967; 193:327-342. [PubMed: 6065881]

12. DeAngelis GC, Ohzawa I, Freeman RD. Depth is encoded in the visual cortex by a specialized receptive field structure. Nature. 1991; 352:156-159. [PubMed: 2067576]

13. Anzai A, Ohzawa I, Freeman RD. Neural mechanisms underlying binocular fusion and stereopsis: position vs. phase. Proc Natl Acad Sci USA. 1997; 94:5438-5443. [PubMed: 9144256]

14. Prince SJ, Pointon AD, Cumming BG, Parker AJ. Quantitative analysis of the responses of V1 neurons to horizontal disparity in dynamic random-dot stereograms. J Neurophysiol. 2002; 87:191-208. [PubMed: 11784742]

15. Haefner RM, Cumming BG. Adaptation to natural binocular disparities in primate V1 explained by a generalized energy model. Neuron. 2008; 57:147-158. [PubMed: 18184571]

16. Hubel DH, Wiesel TN. Binocular interaction in striate cortex of kittens reared with artificial squint. J Neurophysiol. 1965; 28:1041-1059. [PubMed: 5883731]

17. Mitchell, D. The Visual Neurosciencs. Chalupa, LM.; Werner, JS., editors. MIT Press; Cambridge: 2004. p. 189-204.

18. Ohzawa I, Freeman RD. The binocular organization of simple cells in the cat's visual cortex. J Neurophysiol. 1986; 56:221-242. [PubMed: 3746398]

19. Freeman RD, Ohzawa I. Development of binocular vision in the kitten's striate cortex. J Neurosci. 1992; 12:4721-4736. [PubMed: 1464764]

20. Chino YM, Smith EL 3rd, Hatta S, Cheng H. Postnatal development of binocular disparity sensitivity in neurons of the primate visual cortex . J Neurosci. 1997; 17:296-307. [PubMed: 8987756]

21. Maruko I, Zhang B, Tao X, Tong J, Smith EL 3rd, Chino YM. Postnatal development of disparity sensitivity in visual area 2 (V2) of macaque monkeys. J Neurophysiol. 2008; 100:2486-2495. [PubMed: 18753321]

22. Ohki K, Chung S, Ch'ng YH, Kara P, Reid RC. Functional imaging with cellular resolution reveals precise micro-architecture in visual cortex. Nature. 2005; 433:597-603. [PubMed: 15660108]

23. Ohzawa I, DeAngelis GC, Freeman RD. Stereoscopic depth discrimination in the visual cortex: neurons ideally suited as disparity detectors. Science. 1990; 249:1037-1041. [PubMed: 2396096]

24. Bonhoeffer T, Kim DS, Malonek D, Shoham D, Grinvald A. Optical imaging of the layout of functional domains in area 17 and across the area 17/18 border in cat visual cortex. Eur J Neurosci. 1995; 7:1973-1988. [PubMed: 8528473]

25. Ringach DL. On the origin of the functional architecture of the cortex. PLoS ONE. 2007; 2:e251. [PubMed: 17330140]

26. Shimojo S, Silverman GH, Nakayama K. An occlusion-related mechanism of depth perception based on motion and interocular sequence. Nature. 1988; 333:265-268. [PubMed: 3368003]

27. Obermayer K, Blasdel GG, Schulten K. Statistical-mechanical analysis of self-organization and pattern formation during the development of visual maps. Phys Rev A. 1992; 45:7568-7589. [PubMed: 9906831]

28. Swindale NV, Shoham D, Grinvald A, Bonhoeffer T, Hubener M. Visual cortex maps are optimized for uniform coverage. Nat Neurosci. 2000; 3:822-826. [PubMed: 10903576]

29. Yu H, Farley BJ, Jin DZ, Sur M. The coordinated mapping of visual space and response features in visual cortex. Neuron. 2005; 47:267-280. [PubMed: 16039568]

30. Stosiek C, Garaschuk O, Holthoff K, Konnerth A. In vivo two-photon calcium imaging of neuronal networks. Proc Natl Acad Sci USA. 2003; 100:7319-7324. [PubMed: 12777621] 
a

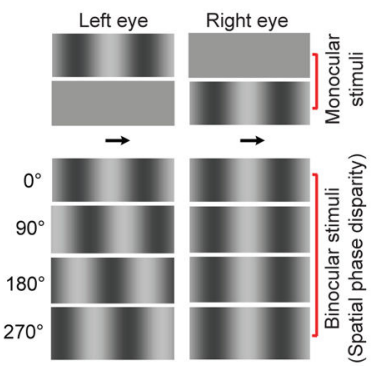

Monoc. Binocular disparity phase

b
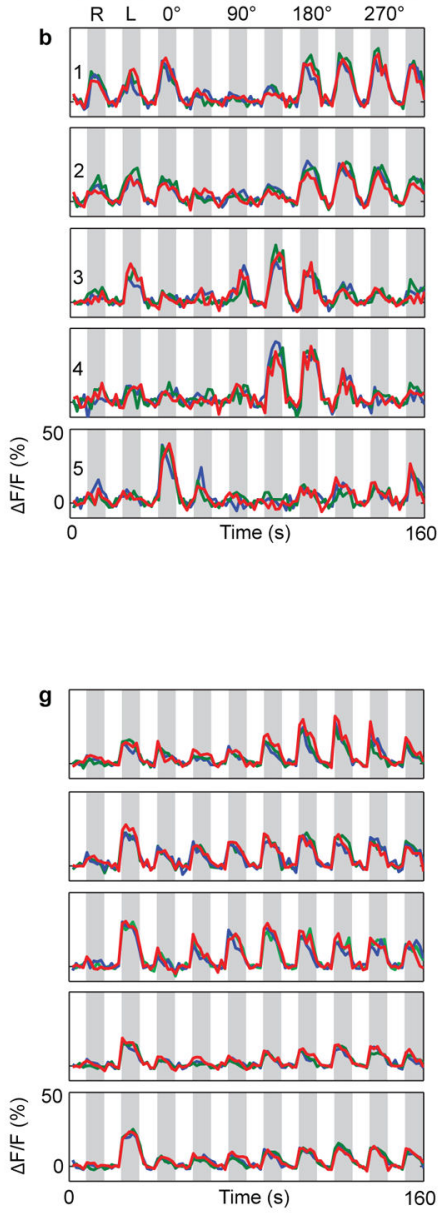
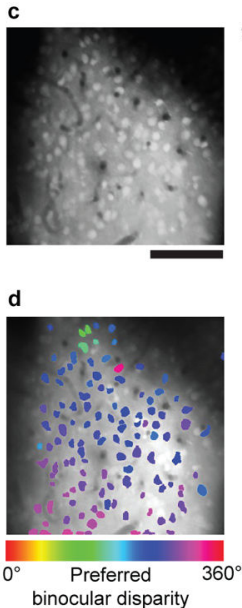

phase

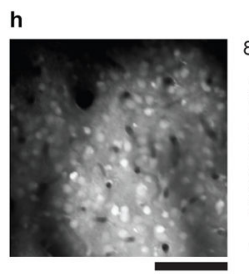

i

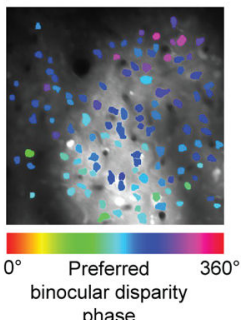

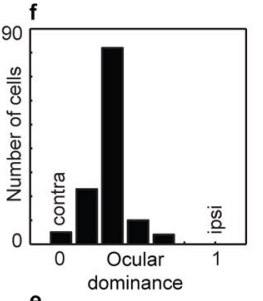
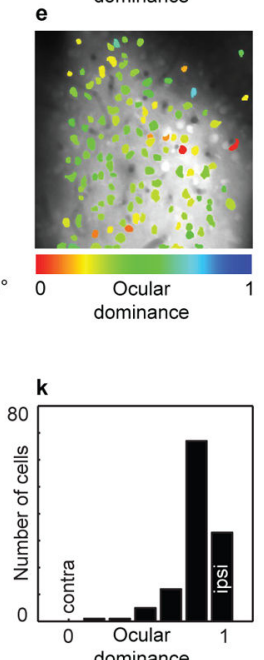

j

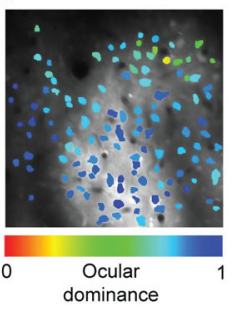

Figure 1.

Single-cell responses and functional maps from two experiments. a, Monocular and binocular stimuli used to obtain maps for ocular dominance and binocular disparity, respectively. Arrows pointing in the same direction denote that the grating stimuli presented to each eye always moved in the same direction during monocular and binocular viewing conditions. b, Time courses for five cells numbered (1-5) from the site shown in c. Three trials are superimposed for each cell. Responses are shown for stimulation to the right eye $(\mathrm{R})$, left eye (L) and then eight inter-ocular spatial phase binocular disparities in $45^{\circ}$ steps. c, Calcium indicator loading in cells $201 \mu \mathrm{m}$ below the pia. d, Cell-based binocular disparity 
map. Only cells significantly tuned for disparity are coloured: $119 / 140$ cells, $P<0.05$, ANOVA across eight disparities. e, Cell-based ocular dominance map. f, Ocular dominance histogram. Data from a second animal (g-k) for which monocular stimuli evoked responses primarily from the ipsilateral eye, but 114 of 124 cells were selectivity tuned for binocular disparity. The preferred orientation for cells at both cortical sites was $45^{\circ}$ from vertical. Scale bars, $100 \mu \mathrm{m}$. 
a
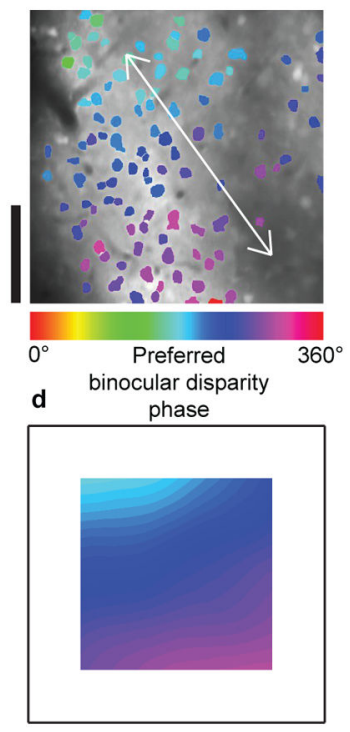

g

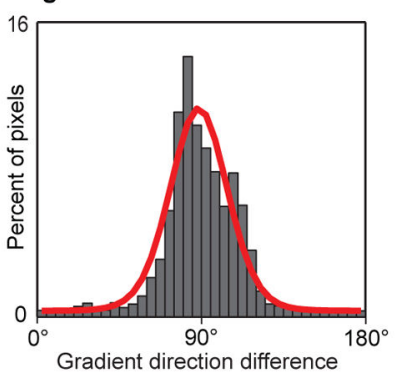

b

e
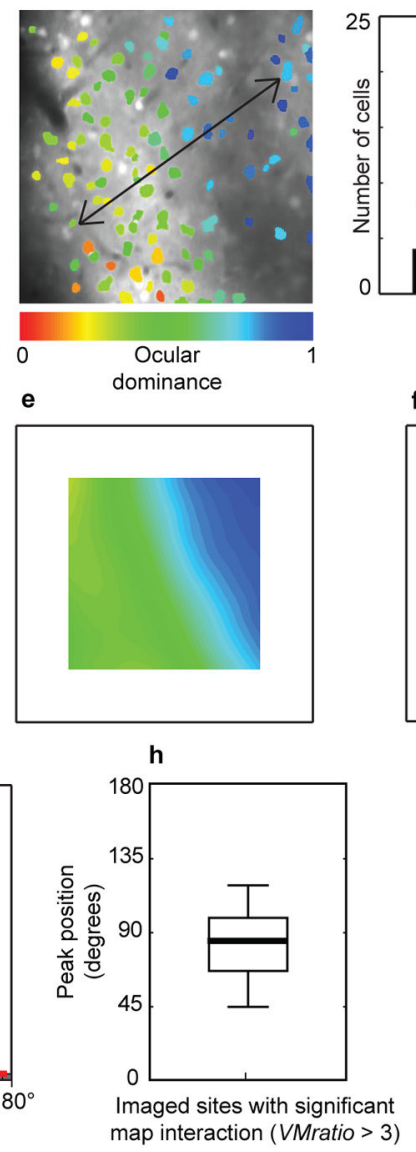

c

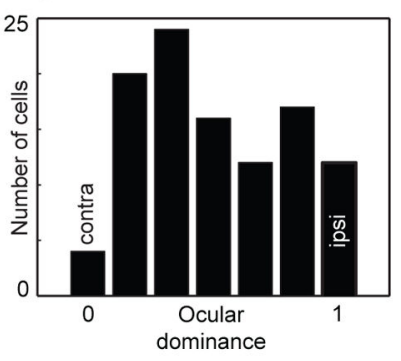

f

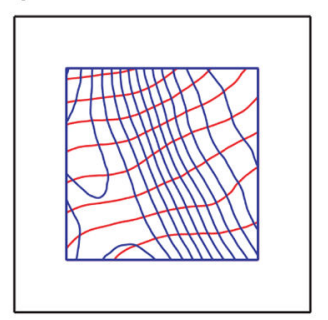

Figure 2.

Orthogonal maps for binocular disparity and ocular dominance when gradients were evident in both maps. a-b, Disparity and ocular dominance cell-based maps from a single imaged site $204 \mu \mathrm{m}$ below the pia. c, Ocular dominance histogram shows that the complete range of ocular dominance indices ( $0-1)$ are represented at this site. The preferred orientation for cells at this site was vertical. d-e, Smoothed disparity and ocular dominance pixel-based maps used in the calculation for the difference in gradient direction for the two maps (g). To avoid artefacts, edges (52 $\mu \mathrm{m}$ on each side) were excluded from the analysis (also see Supplementary Fig. S9). f, Overlay of smoothed disparity and ocular dominance maps, each represented as contour plots (red for disparity, blue for ocular dominance) is indicative of orthogonality. g, Histogram of gradient direction difference for all pixels in the two maps show a peak centred near 90o. Red trace shows curve fitted to the histogram, peak at 880 , confirming orthogonality. $\mathbf{h}$, Range of orthogonality for 8 of 16 imaged areas $(300 \times 300 \mu \mathrm{m}$ each) that had significant interaction. Bold horizontal line represents the median, boxes the $25 \%$ and $75 \%$ quartiles, whiskers the $1 \%$ and $99 \%$ quantiles. Scale bar, $100 \mu \mathrm{m}$. 
a

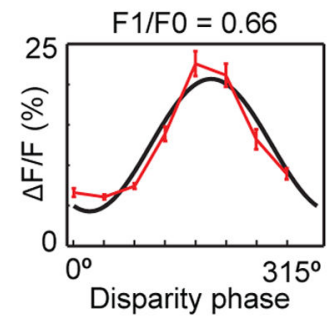

$\mathrm{F} 1 / \mathrm{F} 0=0.43$

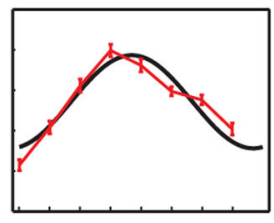

b

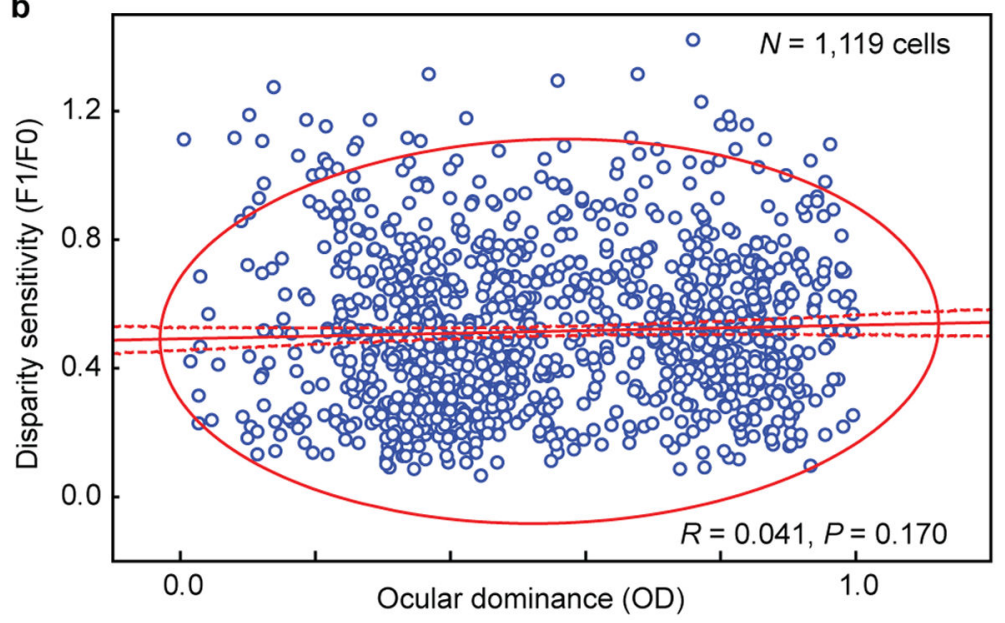

Figure 3.

Relationship between disparity sensitivity and the response to monocular stimuli. a,

Disparity tuning curve for three cells. Data are shown in red, mean \pm s.e.m, for the eight disparities presented. Sine fits are shown in black. The ratio of the amplitude of the sine fit to the measured mean response of the data $(\mathrm{F} 1 / \mathrm{F} 0)$ is a reliable measure of sensitivity to disparity (see Supplementary Fig. S6). b, Disparity sensitivity was uncorrelated with ocular dominance ( $R=0.041, P=0.170, n=1,119$ cells). Dashed lines are $95 \%$ confidence limits for the linear regression and the ellipse is the $95 \%$ prediction interval. 

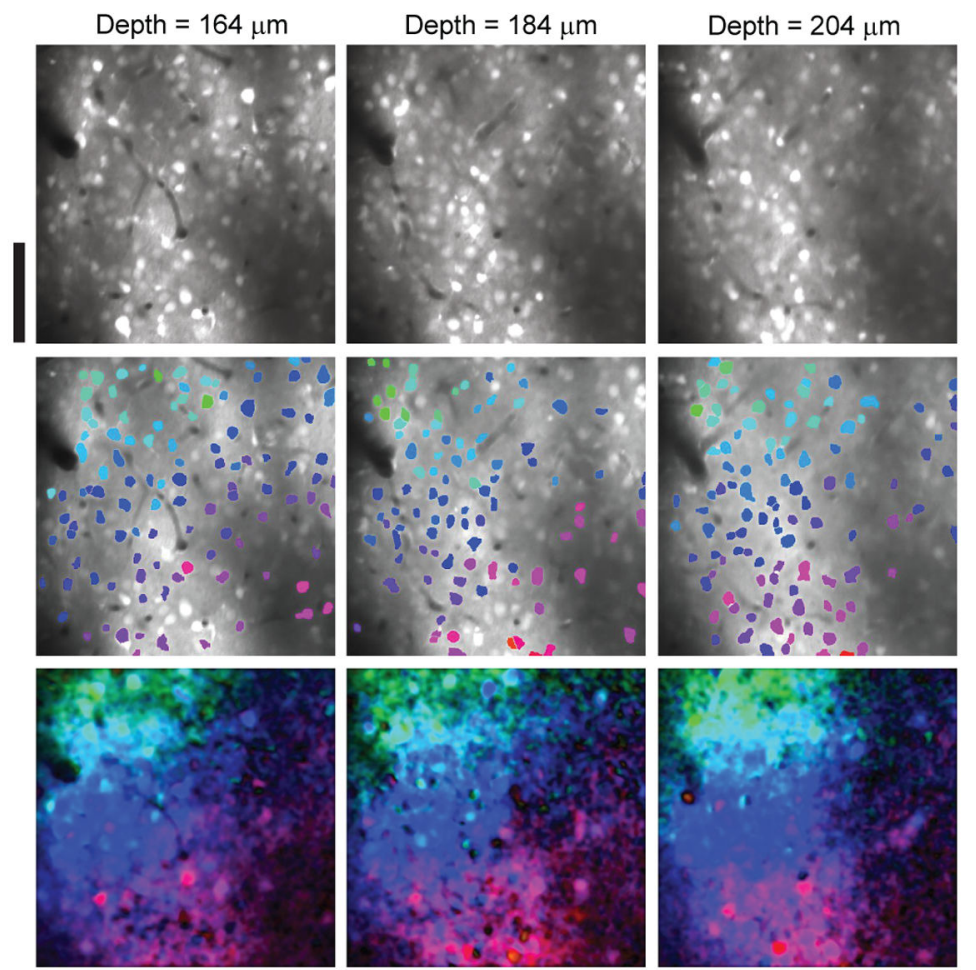

$0^{\circ}$

Preferred binocular disparity phase

$360^{\circ}$

\section{Figure 4.}

Stable functional micro-architecture for binocular disparity. Anatomical images $300 \times 300$ $\mu \mathrm{m}$ (top row), cell-based disparity phase maps (middle row), and pixel-based disparity phase maps (bottom row) obtained at three depths $(164,184$, and $204 \mu \mathrm{m})$ from a single site. Each data set was collected 60-90 minutes apart. The disparity gradient (degrees per $\mu \mathrm{m}$ ) was similar for all three maps (mean \pm s.d. $=0.518 \pm 0.154 ; 0.585 \pm 0.137 ; 0.514 \pm 0.103$ ). The preferred orientation for cells at this site was vertical. An additional data set from this site was collected nearly 12 hours after the first (see Supplementary Fig. S3). Scale bar, $100 \mu \mathrm{m}$. 